\title{
Evidence for a resonant cyclotron line in IGR J16493-4348 from the Swift-BAT hard X-ray survey
}

\author{
A. D’Aì ${ }^{1}$, G. Cusumano ${ }^{2}$, V. La Parola ${ }^{2}$, A. Segreto ${ }^{2}$, T. Di Salvo ${ }^{1}$, R. Iaria ${ }^{1}$, and N. R. Robba ${ }^{1}$ \\ ${ }^{1}$ Dipartimento di Fisica, Università di Palermo, via Archirafi 36, 90123 Palermo, Italy \\ e-mail: dai@fisica.unipa.it \\ 2 INAF, Istituto di Astrofisica Spaziale e Fisica Cosmica, via U. La Malfa 153, 90146 Palermo, Italy \\ Received 6 April 2011 / Accepted 13 June 2011
}

\begin{abstract}
Context. Resonant absorption cyclotron features are a key diagnostic tool to directly measure the strength of the magnetic field of accreting neutron stars. However, typical values of cyclotron features lie in the high-energy part of the spectrum between $20 \mathrm{keV}$ and $50 \mathrm{keV}$, where detection is often damped by the low quality statistics of results derived from single pointed observations.

Aims. We show that long-term monitoring campaign performed with Swift-BAT of persistently, but faint, accreting high-mass X-ray binaries is able to reveal in their spectra the presence of cyclotron features.

Methods. We extracted the average Swift-BAT 15-150 keV spectrum from the 54 months long Swift-BAT survey of the high-mass X-ray source IGR J16493-4348. To constrain the broadband spectrum, we used soft X-ray spectra from Swift-XRT and Suzaku pointed observations.

Results. We model the spectra using a set of phenomenological models usually adopted to describe the energy spectrum of accreting high-mass X-ray binaries. Irrespective of the models we used, we found significant improvements in the spectral fits adding to the models a broad (10 keV width) absorption feature, with best-fitting energy estimate between 30 and $33 \mathrm{keV}$, that we interpret as evidence of a resonant cyclotron absorption feature. We also discuss possible instrumental biases related to the use of Swift-BAT for this kind of study and the statistical method to weight the confidence level of this detection. Correcting for the gravitational redshift of a $1.4 M_{\odot}$ neutron star, the inferred surface magnetic field is $B_{\text {surf }} \sim 3.7 \times 10^{12}$ gauss. The spectral parameters of IGR J16493-4348 fit well with empirical correlations observed when the whole sample of high-mass binaries with detected cyclotron features is considered.
\end{abstract}

Key words. magnetic fields - line: profiles - X-rays: binaries - X-rays: individuals: IGR J16493-4348

\section{Introduction}

The Burst Alert Telescope (BAT, Barthelmy et al. 2005) on board Swift (Gehrels et al. 2004) has been performing a continuous monitoring of the sky in the hard X-ray energy range $(15-150 \mathrm{keV})$ since November 2004. The telescope, thanks to its large field of view (1.4 steradian half-coded) and its pointing strategy, covers a fraction of between $50 \%$ and $80 \%$ of the sky every day. This has allowed the detection of many of the newly high-mass X-ray binaries (HMXBs, e.g., Cusumano et al. 2010b) detected by INTEGRAL and the collection of their longterm light curves and spectra. The long and continuous monitoring of these sources allows us to investigate the intrinsic emission variability, to search for long-term periodicities (orbital periods) and to discover eclipse events. The role of SwiftBAT (hereafter simply BAT) is therefore fundamental to unveiling the nature and the geometry of these binary systems. Moreover, BAT collects their long-term averaged energy spectra in the 15.0-150 keV band. For HMXBs, key resonant cyclotron scattering features (CRSFs) are usually observed in this part of the X-ray spectrum (Heindl et al. 2004).

In this paper, we analyse the soft and hard X-ray data collected by BAT, INTEGRAL, and pointed Suzaku and Swift-XRT observations on IGR J16493-4348. This source was discovered by INTEGRAL in 2004 (Bird et al. 2004) and initially associated with the radio pulsar PSR J1649-4349 because of a spatial coincidence. A later INTEGRAL observation with a deep exposure allowed to reduce the positional uncertainty and reject the pulsar association (Grebenev et al. 2005). A followup observation with Chandra found a soft X-ray counterpart at a position of $\mathrm{RA}(\mathrm{J} 2000)=16^{\circ} 49^{\prime} 26.92^{\prime \prime}, \operatorname{Dec}(\mathrm{J} 2000)=$ $-43^{\circ} 49^{\prime} 8.96^{\prime \prime}$ (Kuiper et al. 2005) allowing the association with the infrared counterpart 2MASS J1642695-4349090, a B0.5 Ib supergiant. Nespoli et al. (2010) performed $K$-band spectroscopy of the inferred counterpart, confirming the B0.5-1 Ia-Ib companion spectral type. In addition, the infrared extinction translated into the equivalent hydrogen column density, was estimated to be $(2.92 \pm 1.96) \times 10^{22} \mathrm{~cm}^{-2}$, a value that is lower than the X-ray absorption, thus indicating that part of the X-ray absorption may be local to the compact object. Cusumano et al. (2010a) found a periodicity of $6.732 \pm 0.002 \mathrm{~d}$, which is interpreted as the orbital period of the system. The folded light curve shows the presence of an eclipse, lasting $\sim 12 \%$ of the $P_{\text {orb }}$. Assuming a $1.4 M_{\odot}$ neutron star (NS) and $32 R_{\odot}$ for the companion star, the system has most likely a low eccentricity value $(e \leq 0.15)$. Corbet et al. (2010) found in RXTE observations evidence for a shorter periodicity at $1069 \pm 7 \mathrm{~s}$, which was interpreted as the NS spin period.

A spectral analysis using non-simultaneous data from Swift-XRT and INTEGRAL (Hill et al. 2008) showed that the broadband X-ray spectrum could be fitted well by an absorbed $\left(N_{\mathrm{H}}=5.6 \times 10^{22} \mathrm{~cm}^{-2}\right)$ power law $(\Gamma=0.6 \pm 0.3)$ with a highenergy cut-off at $\sim 17 \mathrm{keV}$. Morris et al. (2009) analysing Suzaku data of IGR J16493-4348, proposed an alternative modelling of the X-ray spectrum using a partial covering component multiplied by a simple power-law. The covering fraction of the total 
emission was estimated to be $0.62 \pm 0.07$, while the local value of the $N_{\mathrm{H}}$ was estimated to be $\sim 30 \times 10^{22} \mathrm{~cm}^{-2}$.

\section{Data reduction}

The raw BAT survey data of the first 54 months of the Swift mission were retrieved from the HEASARC public archive ${ }^{1}$ and processed with a dedicated software (Segreto et al. 2010) that performs screening, mosaicking, and source detection on BAT data and produces spectra and light curves for any given sky position. The light curve of IGR J16493-4348 was extracted in the $15-150 \mathrm{keV}$ energy range with the maximum available time resolution $(\sim 300 \mathrm{~s})$. The spectrum was obtained by extracting the source count rates in 16 energy bands and analysed using the BAT spectral redistribution matrix ${ }^{2}$. The last three energy channels (100-150 keV range) were rebinned into a single channel to ensure a signal-to-noise ratio above 3 standard deviations (hereafter $\sigma$ ). The source is detected at a significance level of $\sim 21 \sigma$. The average count rate in the BAT light curve is $1.03 \times 10^{-4}$ count s$^{-1}$ pixel $^{-1}$. When considering the light curve at the highest resolution, the maximum deviation from the average rate is about $7 \sigma$, corresponding to an increase in the rate of a factor of $\sim 20$. Although the count rate indicates brightness variations in the accreting source, we found no evidence of spectral shape variability, when spectra collected on a one-month time span were fitted using a simple power-law model, with photon indices consistent with the average value within the errors. The $15-50 \mathrm{keV}$ and $50-150 \mathrm{keV}$ time-averaged fluxes are $(3.0 \pm 0.3) \times 10^{-11} \mathrm{erg} \mathrm{cm}^{-2} \mathrm{~s}^{-1}$ and $\left(1.4_{-0.3}^{+0.1}\right) \times 10^{-11} \mathrm{erg} \mathrm{cm}^{-2} \mathrm{~s}^{-1}$, respectively.

We also analysed INTEGRAL-ISGRI (hereafter simply ISGRI) long-term data obtained from the Integral high-level products archive HEAVENS ${ }^{3}$. ISGRI data were used in the $15-150 \mathrm{keV}$ energy range.

To have the necessary coverage of the soft X-ray band, we used data of the Swift-XRT (hereafter simply XRT, Burrows et al. 2005) observation performed on 2006 March 11 (Obs.ID 00030379002), for a total exposure time of $5.6 \mathrm{ks}$ and a Suzaku (Mitsuda et al. 2007) observation performed on 2006 October 10 (Obs.ID 401054010, see Morris et al. 2009, for further details).

The XRT data were processed with standard procedures (XRTPIPELINE v.0.12.4), filtering and screening criteria, using FTOOLS in the HEASOFT package ( $\mathrm{v}$ 6.8). The source was observed in photon counting mode (Hill et al. 2004). We adopted standard grade filtering $0-12$. The source events for spectral analysis were extracted from a circular region of 20 pixel radius (1 pixel $\left.=2.36^{\prime \prime}\right)$ centred on the source position as determined with XRTCENTROID. The background was extracted from an annular region centred on the source with radii of 70 and 130 pixels, respectively. The spectrum was analysed using ancillary response files generated with XRTMKARF and spectral redistribution matrix v011.

We extracted Suzaku scientific data (Obs.ID 401054010) from clean events reprocessed with the 2.0.6.13 standard pipeline procedure. We obtained four spectra from the X-ray Imaging Spectrometer (XIS(0-4), Koyama et al. 2007). Spectra from the front-illuminated CCD (XIS0, XIS2 and XIS3) were combined into a single spectrum (XIS023) using the

\footnotetext{
${ }^{1}$ http://heasarc.gsfc.nasa.gov/docs/archive.html

${ }^{2}$ http://heasarc.gsfc.nasa.gov/docs/heasarc/caldb/

data/swift/bat/index.html

3 http://www.isdc. unige.ch/heavens/
}

addascaspec script, while the XIS1 spectrum was used in the analysis as a different dataset.

The source extraction region was centred on the CHANDRA source position with a radius of $140^{\prime \prime}$. We used a region of the same size, with no apparent contaminating sources, to extract the background spectrum. Data from the HXD/PIN instrument (Takahashi et al. 2007) were obtained using the hxdpinxbpi script. The HXD/PIN background is the sum of a time-variable instrumental background (non-X-ray background, $\mathrm{NXB}$ ) induced by cosmic rays and trapped charged particles in the satellite orbit and the intrinsic cosmic background (cosmic $\mathrm{X}$-ray background, CXB). We used the 2.0 version of the tuned NXB model spectrum released by the HXD instrument team (LCFITDT model, Fukazawa et al. 2009) and the CXB spectrum obtained from the Boldt (1987) X-ray background model convolved with the PIN response for the flat emission distribution at the epoch of this observation. Given the lack of local narrow features, spectra from XIS and HXD/PIN were coarsely rebinned into a spectrum of 84 (21) energy channels for the XIS023 (XIS1) and nine energy channels for the HXD/PIN. This choice provides a $\mathrm{S} / \mathrm{N}$ of above three for each energy channel. XIS data are background dominated below $2 \mathrm{keV}$ and above $10 \mathrm{keV}$, so that these channels were not used in the spectral analysis. The HXD/PIN spectrum was used in the $12.0-40.0 \mathrm{keV}$ energy range.

Spectral fits were performed using XSPEC v.12.6.0. Errors are at $90 \%$ confidence level, if not stated otherwise.

\section{Spectral analysis}

BAT data were collected during the monitoring campaign from 2004 December to 2009 May, ISGRI data from 2003 January to 2009 April, and both sets of data track, within the same temporal window, the long-term average hard X-ray emission. Swift-XRT and Suzaku observations, as indicated in the previous section, are pointed observations taken at different times. To constrain the broadband spectrum, we distinguished, therefore, the two soft X-ray observations performed with XRT and Suzaku. We hereafter define spectrum 1 to be the spectrum containing the datasets XRT, BAT, and ISGRI. For spectrum 2, the spectrum was compiled from the datasets Suzaku (XIS1, XIS023 and HXD/PIN), BAT, and ISGRI.

We adopted an inter-calibration multiplicative constant to take into account flux uncertainties in the response of the different instruments. A common fit to the ISGRI and BAT data does not show evidence any systematic flux mismatch. Data could satisfactorily be fitted using a power-law model and the residuals did not show any systematic difference in the two datasets. A calibration constant between the datasets (fixed to one for the BAT data and allowed to vary for the ISGRI data) was found to be consistent with one. Therefore, for the following analysis we fixed the inter-calibration factor between these two instruments to one.

For spectrum 1, the constant was fixed to one for the XRT dataset and allowed to vary for the BAT/ISGRI datasets $\left(C_{\text {bat }}\right)$. For spectrum 2, the constant was fixed to 1 for the XIS023 spectrum and allowed to vary for the HXD-PIN dataset $\left(C_{\mathrm{hxd}}\right)$, the XIS1 dataset $\left(C_{\text {xis } 1}\right)$, and the BAT/ISGRI dataset $\left(C_{\text {bat }}\right)$. The $1-10 \mathrm{keV}$ absorbed/unabsorbed flux was $(5.26 \pm 0.08 / 8.15 \pm$ $0.15) \times 10^{-11} \mathrm{erg} \mathrm{cm}^{-2} \mathrm{~s}^{-1}$ for the Swift-XRT observation, whereas it was $(1.36 \pm 0.01 / 2.35 \pm 0.05) \times 10^{-11} \mathrm{erg} \mathrm{cm}^{-2} \mathrm{~s}^{-1}$ during the Suzaku observation (errorbars take also into account the uncertainty associated with different models of the spectra). 
To model the data, we employed the three most commonly used models to fit broadband spectra of accreting high-mass X-ray binaries (see Makishima et al. 1999, for a comprehensive discussion of the models): a cut-off power law model (model cutoffpl), a power-law with a Fermi-Dirac cut-off (model fdco), the negative-positive power-law model (model npex, with the positive photon index fixed to +2 ). The cutoffpl model has three main parameters: the photon-index $(\Gamma)$, the cutoff energy ( $\left.E_{\text {cut }}\right)$, and the normalization of the power-law $\left(N_{\text {po }}\right)$. The fdco model has one more parameter, the folding energy of the power-law $\left(E_{\mathrm{fol}}\right)$. The npex is basically the sum of two power-laws with a common exponential cut-off.

We did not use the partial covering model of a power-law spectrum as in Morris et al. (2009), because this model is not physically consistent with the nature of IGR J16493-4348, as the $6.732 \mathrm{~d}$ orbital period and the $1069 \mathrm{~s}$ spin period place unambiguously IGR J16493-4348 in the wind-fed HMXB zone of the $P_{\text {orb }}-P_{\text {spin }}$ diagram (also known as the Corbet diagram, Corbet 1984).

\subsection{Spectrum 1}

In the first column of Table 1, we present the best-fit results adopting our set of spectral models for the spectrum 1 datasets.

The absorbed power-law with a high energy exponential cutoff gives a column density $N_{\mathrm{H}}=(7.0 \pm 1.2) \times 10^{22} \mathrm{~cm}^{-2}$, $\Gamma=0.7 \pm 0.3$ and a cut-off energy $E_{\text {cut }}=20 \pm 5 \mathrm{keV}$. This model already proposed in Hill et al. (2008) is a more accurate representation of the data with respect to the more sophisticated fdco model, but we found that the npex model provides still a better fit to the data, even if the steepness of the power-law, the cut-off energy and the $N_{\mathrm{H}}$ are consistent in the two models. Irrespective of the model adopted, broad residuals were evident between 30 and $40 \mathrm{keV}$ (see Fig. 1). Inspecting the data, we noted that the residuals were mostly driven by the residuals of the BAT data, because of the higher $\mathrm{S} / \mathrm{N}$ of these data in the hard X-ray band.

We tentatively identified the shape of the residuals with a broad feature in absorption and repeated the fits introducing a cyclotron absorption feature (cyclabs component, second column in Table 1), which is expressed according to the formula

$C Y C L A B S(E)=D_{\mathrm{c}} \frac{\left(W_{\mathrm{c}} E / E_{\mathrm{c}}\right)^{2}}{\left(E-E_{\mathrm{c}}\right)^{2}+W_{\mathrm{c}}^{2}}$,

where $E_{\mathrm{c}}, D_{\mathrm{c}}$, and $W_{\mathrm{c}}$ are the cyclotron energy, depth, and width respectively (Makishima et al. 1990). We assume that the broad feature constitutes the fundamental and, given the low $\mathrm{S} / \mathrm{N}$ at higher energies, we neglect higher harmonics.

The line parameters could not be all adequately constrained. We found that the width of the line strongly correlated with the cut-off energy of the continuum models, resulting in a large uncertainty. We, therefore, kept this parameter frozen to the value of $10 \mathrm{keV}$, as this was the most reasonable value that provided the lowest $\chi^{2}$ in all the models adopted. This choice is later also discussed a posteriori. The energy of the line, for the npex best-fitting model is $33 \pm 4 \mathrm{keV}$, while the depth of the line is $0.5 \pm 0.2$. Similar values were found also when adopting the other two models, implying that the line shape is weakly correlated with the assumed broadband continuum shape. The addition of this component significantly improved the fit (reduced $\chi^{2}$ 1.16 compared to a value of 1.32 without the line).

To fit CRSFs, a Gaussian absorption model (gabs in Xspec) has also been often used (Coburn et al. 2002). Replacing the
Table 1. Spectral fitting results.

\begin{tabular}{|c|c|c|c|c|}
\hline & \multicolumn{2}{|c|}{ Spectrum1 } & \multicolumn{2}{|c|}{ Spectrum2 } \\
\hline & No cyc & $+C y c$ & No cyc & $+C y c$ \\
\hline & \multicolumn{4}{|c|}{ cutoffpl } \\
\hline$N_{\mathrm{H}}$ & $7.0_{-1.2}^{+1.5}$ & $5.5_{-0.8}^{+1.5}$ & $7.7_{-0.5}^{+0.4}$ & $7.5 \pm 0.4$ \\
\hline$\Gamma$ & $0.7 \pm 0.3$ & $0.4_{-0.2}^{+0.4}$ & $1.1 \pm 0.1$ & $1.13 \pm 0.12$ \\
\hline$N_{\mathrm{po}}\left(10^{-3}\right)$ & $5.4_{-2.0}^{+4}$ & $2.7_{-0.8}^{+2.5}$ & $2.5 \pm 0.5$ & $2.4 \pm 0.4$ \\
\hline$E_{\text {cut }}(\mathrm{keV})$ & $20 \pm 5$ & $19_{-2}^{+6}$ & $24_{-3}^{+4}$ & $27_{-5}^{+6}$ \\
\hline$C_{\text {bat }}$ & $0.23_{-0.05}^{+0.08}$ & $0.18_{-0.03}^{+0.07}$ & $1.18 \pm 0.14$ & $1.28 \pm 0.11$ \\
\hline$C_{\mathrm{xis} 1}$ & & & $1.09 \pm 0.03$ & $1.09 \pm 0.03$ \\
\hline$C_{\mathrm{hxd}}$ & & & $0.92 \pm 0.13$ & $0.92 \pm 0.15$ \\
\hline$E_{\text {cyc }}(\mathrm{keV})$ & & $32 \pm 4$ & & $30 \pm 5$ \\
\hline$D_{\mathrm{c}}$ & & $0.6 \pm 0.2$ & & $0.45 \pm 0.17$ \\
\hline \multirow[t]{2}{*}{$\chi_{\text {red }}^{2}$ (d.o.f.) } & 1.41 (106) & 1.18 (104) & 1.24 (133) & $1.12(131)$ \\
\hline & \multicolumn{4}{|c|}{ fdco } \\
\hline$N_{\mathrm{H}}$ & $7.3_{-1.3}^{+1.5}$ & $6.0_{-1.2}^{+1.1}$ & $8.0 \pm 0.6$ & $7.8 \pm 0.6$ \\
\hline$\Gamma$ & $0.9 \pm 0.3$ & $0.6 \pm 0.3$ & $1.28 \pm 0.12$ & $1.24 \pm 0.11$ \\
\hline$N_{\text {po }}\left(10^{-3}\right)$ & $12_{-5}^{+10}$ & $5.2 \pm 0.8$ & $5.8 \pm 0.6$ & $5.3 \pm 1.1$ \\
\hline$E_{\mathrm{fol}}(\mathrm{keV})$ & $18_{-3}^{+5}$ & $19_{-3}^{+4}$ & $22_{-2}^{+3}$ & $24_{-3}^{+4}$ \\
\hline$E_{\text {cut }}(\mathrm{keV})$ & $<7.4$ & $<20$ & $<8$ & $<22$ \\
\hline$C_{\text {bat }}$ & $0.22_{-0.05}^{+0.08}$ & $0.18 \pm 0.05$ & $1.13 \pm 0.11$ & $1.24_{-0.17}^{+0.22}$ \\
\hline$C_{\text {xis1 }}$ & & & $1.10 \pm 0.03$ & $1.10 \pm 0.03$ \\
\hline$C_{\mathrm{hxd}}$ & & & $0.90 \pm 0.13$ & $0.91 \pm 0.15$ \\
\hline$E_{\text {cyc }}(\mathrm{keV})$ & & $32.4 \pm 3.5$ & & $31 \pm 5$ \\
\hline$D_{\mathrm{c}}$ & & $0.6 \pm 0.2$ & & $0.50 \pm 0.17$ \\
\hline \multirow[t]{2}{*}{$\chi_{\text {red }}^{2}$ (d.o.f.) } & 1.48 (105) & $1.21(103)$ & 1.33 (132) & 1.17 (130) \\
\hline & \multicolumn{4}{|c|}{ npex } \\
\hline$N_{\mathrm{H}}$ & $7.3_{-1.3}^{+1.1}$ & $5.8_{-1.0}^{+1.2}$ & $7.7 \pm 0.6$ & $7.5 \pm 0.5$ \\
\hline$\Gamma$ & $0.81_{-0.3}^{+0.15}$ & $0.45 \pm 0.3$ & $1.05_{-0.6}^{+0.1}$ & $1.01 \pm 0.16$ \\
\hline$E_{\text {cut }}(\mathrm{keV})$ & $15.3_{-2.7}^{+0.7}$ & $16_{-4}^{+6}$ & $16_{-2}^{+4}$ & $17_{-1}^{+6}$ \\
\hline$N_{p}^{a}\left(10^{-8}\right)$ & $6.4_{-4.7}^{+1.7}$ & $<70$ & $1.3_{-0.9}^{+0.2}$ & $1.0_{-0.9}^{+0.5}$ \\
\hline$N_{n}^{b}\left(10^{-3}\right)$ & $6.1_{-2.4}^{+2.8}$ & $3.1 \pm 0.1$ & $2.4 \pm 0.5$ & $2.2 \pm 0.4$ \\
\hline$C_{\text {bat }}$ & $0.28_{-0.08}^{+0.06}$ & $0.20 \pm 0.06$ & $1.35 \pm 0.14$ & $1.41 \pm 0.18$ \\
\hline$C_{\text {xis1 }}$ & & $1.10 \pm 0.03$ & $1.10 \pm 0.03$ & \\
\hline$C_{\text {hxd }}$ & & $1.06 \pm 0.15$ & $1.04 \pm 0.16$ & \\
\hline$E_{\text {cyc }}(\mathrm{keV})$ & & $33 \pm 4$ & & $31_{-12}^{+8}$ \\
\hline$D_{\mathrm{c}}$ & & $0.5 \pm 0.2$ & & $0.30_{-0.14}^{+0.19}$ \\
\hline$\chi_{\text {red }}^{2}$ (d.o.f.) & $1.32(105)$ & $1.16(103)$ & $1.16(132)$ & $1.10^{-0.14}(130)$ \\
\hline
\end{tabular}

Notes. (a) Normalization of the power-law with positive index. (b) Normalization of the power-law with negative index.

cyclabs with the gabs model, did not significantly change the conclusions of our analysis. We again found a tight correlation between the depth and $\sigma$ of the line. The best-fit position of the line varied, according to the model adopted, between $31 \mathrm{keV}$ and $35 \mathrm{keV}$. Keeping the line $\sigma$ frozen at $5 \mathrm{keV}$, the best-fit depth of the line was found to be between 1 and 5 . Relative errors in the best-fit parameters were of the same order as the corresponing cyclabs analogues. The $\chi^{2}$ values were found to be marginally poorer than for the models with the cyclabs component. The best-fit value of $C_{\text {bat }}$ is $\sim 0.2$. We verified that this value is consistent with the intensity of the BAT light curve during the XRT observation, a factor $\sim 4$ higher than the average source intensity along the 54 months of monitoring. We show in Fig.1 data of spectrum 1 and residuals for the models of first/second columns of Table 1. 

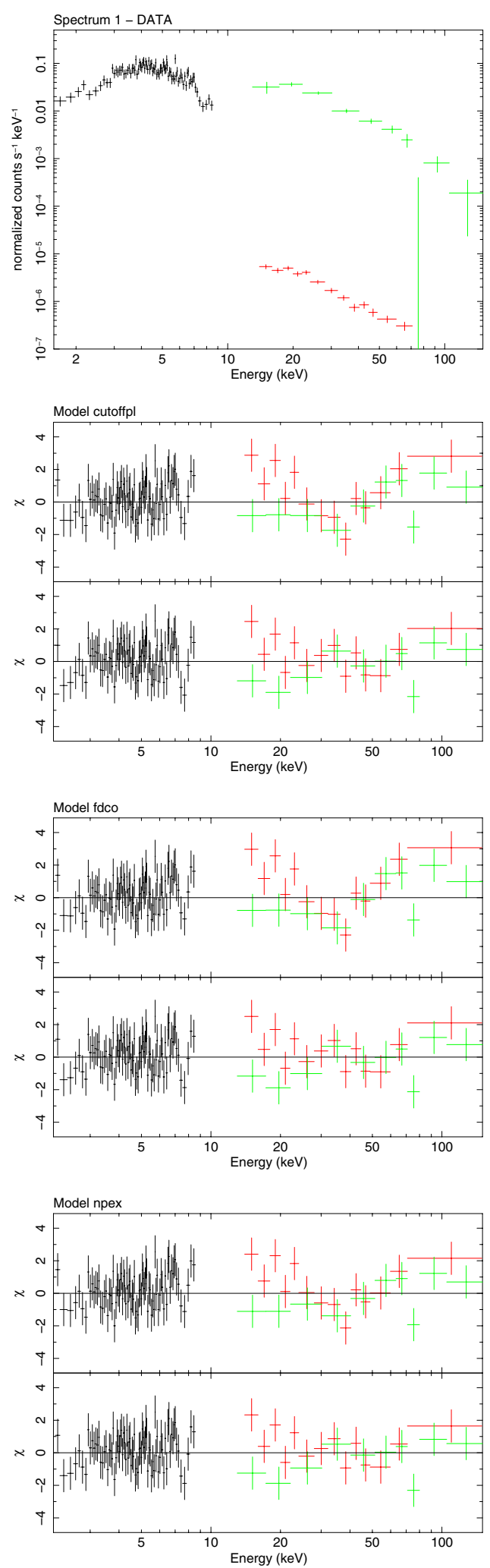

Fig. 1. Upper panel: datasets that compose the spectrum 1 analysis. Black data: XRT spectrum; red data: BAT spectrum; green data: ISGRI spectrum. Lower panels: residuals in units of $\sigma$ for the continuum models of Table 1; upper mid-panels: only continuum, lower mid-panels: continuum with the addition of a cyclotron absorption feature at $\sim 31$ $\mathrm{keV}$.

\subsection{Spectrum 2}

We used the pointed Suzaku observation to perform a complementary analysis, checking how a different soft X-ray spectrum could affect the shape of the hard X-ray spectrum. The analysis was performed in analogy with the steps described for spectrum 1. The third and fourth column of Table 1 show the results of the spectral fitting for the three models, with and without the addition of the cyclotron line. The results are in close agreement with those obtained for spectrum 1 and we find again that the npex model has the lowest reduced $\chi^{2}$. For this model, the normalisation constant $C_{\text {bat }}$ is $\sim 1.4$, which is about six times higher than for spectrum 1; the observed flux during the Suzaku observation is, in fact, about four times fainter than for XRT observation. Despite the flux difference between the XRT and the Suzaku observations, no statistically significant variation in the spectral shape of the emission is, however, observed with consistent best-fit values for the two spectra.

The best fit to the continuum emission (npex model) gave a reduced $\chi^{2}=1.16$ (132 d.o.f.), while the model with the cyclabs component gave a reduced $\chi^{2}=1.10$ (130 d.o.f.). The line shape is found to depend only marginally on the soft X-ray band. The cyclotron line parameters are, however, more poorly constrained than for spectrum 1, although the best-fit model paramenters appear to closely agree. We show in Fig. 2 data of spectrum 2 and residuals for the models of third and fourth column of Table 1.

\subsection{Calibration uncertainties in the BAT instrument}

Because the detection of the cyclotron features is mostly pivoted by the BAT high-energy data, we checked for possible biases in the analysis due to incorrect background subtraction or systematics in the BAT response matrix ${ }^{4}$ by comparing the extracted spectrum of IGR J16493-4348 with the spectra of nearby X-ray sources having similar spectral shape.

We selected from the 54-month BAT catalogue the three HMXBs closest to IGR J16493-4348: IGR J16393-4643, IGR J16418-4532, and AX J1700.2-4220. We extracted the BAT long-term spectra and used pointed Swift-XRT and XMM-Newton (Epic-PN spectrum for AX J1700.2-4220) observations to obtain their broadband $\mathrm{X}$-ray spectra. The spectra of IGR J1639.1-4641 and AX J1700.2-4220 could be closely fitted using a cut-off power-law model, while the spectrum of IGR J16418-4532 required a npex model to be satisfactorily fitted. In each case, the BAT data did not show any structure in the residuals in the $30-40 \mathrm{keV}$ range. Because of the similarity in the shape of the continuum emission, the common background estimate, and the comparable statistics in the BAT data among all the sources of this sample and IGR J16493-4348, we conclude that the residuals shown in the spectrum of IGR J16493-4348 can be attributed to neither the calibration uncertainties of the BAT instrument nor an incorrect estimate, or subtraction, of the X-ray background.

\subsection{A method for testing the significance of the detection}

To estimate the statistical significance of the residuals present in the BAT data of IGR J16493-4348, we adopted the following procedure (see also Suchy et al. 2011 for a similar approach):

- we assumed the best-fit npex model for spectrum 1 in Table 1 without the cyclotron line as the null-hypothesis model;

\footnotetext{
${ }^{4}$ http://swift.gsfc.nasa.gov/docs/swift/analysis/ bat_digest.html
} 

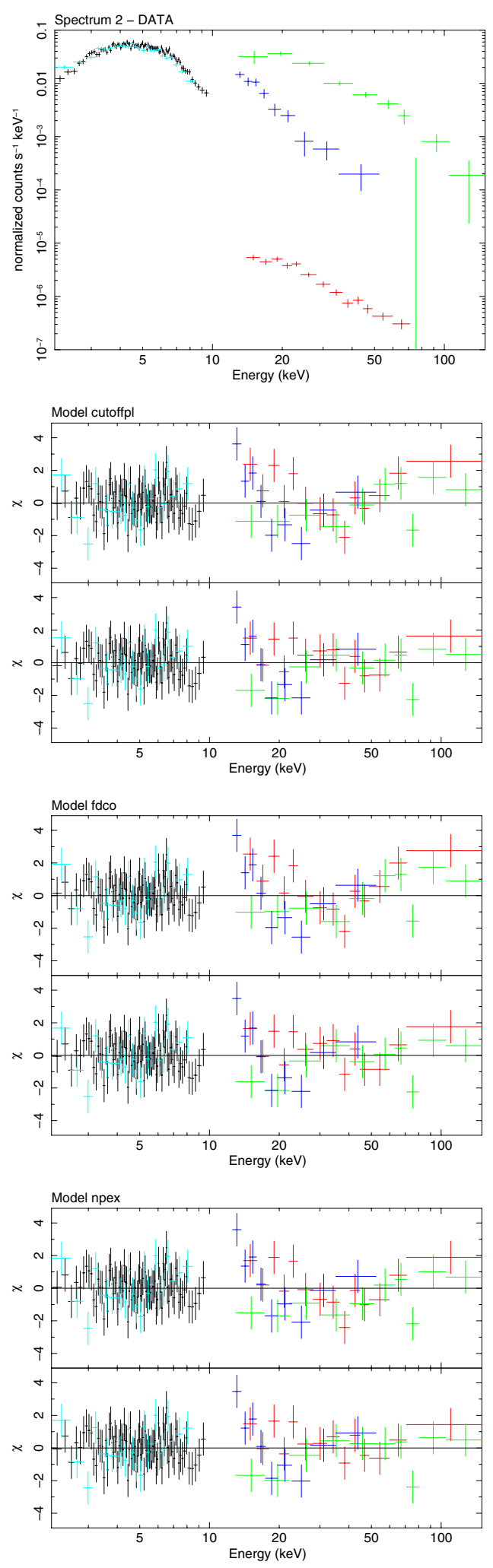

Fig. 2. Upper panel: datasets that compose the spectrum 2 analysis. Black data: XIS023 spectrum; red data: BAT spectrum; green data: ISGRI spectrum; blue data: Suzaku/HXD-PIN spectrum; light blue: XIS0 spectrum. Lower panels: residuals in units of $\sigma$ for the continuum models of Table 1; upper mid-panels: only continuum, lower midpanels: continuum with the addition of a cyclotron absorption feature at $\sim 31 \mathrm{keV}$.

- we simulated accordingly a fake BAT spectrum with the same $\mathrm{S} / \mathrm{N}$ of the real BAT spectrum;
- we again fitted the datasets of spectrum 1 (real data and simulated BAT spectrum) using the npex model with and without the addition of an absorption cyclotron line (width constrained at $10 \mathrm{keV}$, with line energy and optical depth as free parameters);

- we calculated the difference in $\Delta \chi^{2}$ for the two best-fit models: npex and npex+cyclabs;

- we repeated this procedure for an other 10000 simulated BAT spectra;

- we plotted with histograms the $\Delta \chi^{2}$ difference versus the number of spectra;

- we repeated the same procedure for the spectrum 2 datasets.

In Fig. 3, we show the results of our method. In 10000 spectra we did not obtain any difference in the $\Delta \chi^{2}$ as the ones reported in Table 1, both for spectrum 1 (observed $\Delta \chi^{2}=19$ ) and for Spectrum 2 (observed $\Delta \chi^{2}=10$ ) datasets. The shape of the distribution is different in the two cases: the XRT data constrain more weakly the broadband shape, and the addition of a broad feature in absorption in most cases improves the fit. In contrast, the Suzaku data are less affected by fitting improvements even for the addition of a broad, continuum-like, feature. This is expected since the statistical weight of the Suzaku XIS ( $\sim 20000$ counts in the XIS spectra versus the $\sim 2000$ counts of the XRT spectrum) data more strongly pivot the overall determination of the continuum shape and the $\chi^{2}$ result. At the same time, tighter constraints of the continuum shape ensure that strong effects of random fluctuations such as artifacts on the fitting, are less probable. We note, therefore, that the detection level as estimated from the difference in the $\chi^{2}$ of the two models (with and without the addition of the cyclotron absorption feature) in spectrum 2 real data, although in absolute value less than what found for spectrum 1, is consistent and in agreement with the detection level obtained from Spectrum 1 data. Moreover, we estimated this detection level for the model (npex) that provided the lowest improvements to the addition of this features; the other spectral models would have given a much higher detection confidence. From the generation of 10000 simulated spectra, no random fit improvement of the same order, or greater, than the one we obtained from real data was obtained for both datasets, hence we estimate a detection level corresponding to at least $\sim 3.9 \sigma$ for the presence of a cyclotron line in our fits.

\section{Discussion}

There are 15 HMXBs that clearly display CRSFs signatures in their spectra. This number represents about $10 \%$ of the total population of HMXBs detected in our Galaxy (Reig 2011) and, despite the considerable amount of observing time dedicated by modern and past X-ray observatories, to this class of sources, there have not been a substantial number of new detections of CRSFs in the past decade.

We have presented new results based on the analysis of the data collected by BAT during the first 54 months of the Swift mission for a persistent sgHMXB IGR J16493-4348. We have shown that the long-term hard X-ray spectra acquired by BAT enables us to detect CRSFs in this kind of sources. This new approach relies on a series of assumptions and caveats that we now discuss.

The broadband spectrum, modelled with a positive-negative cut-off power-law model provides the statistically most favoured description of the data in the examined datasets. The residuals in the BAT energy range show the presence of an absorption feature between $\sim 28$ and $\sim 38 \mathrm{keV}$. The feature does not depend strongly 

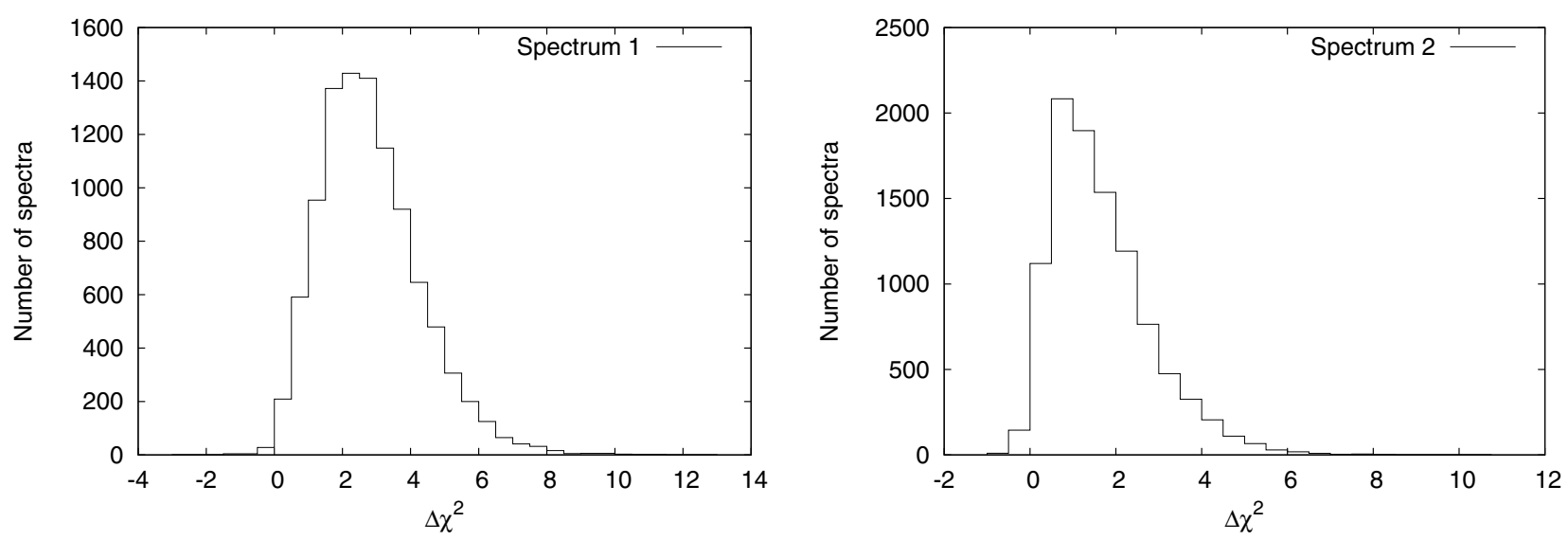

Fig. 3. Histograms of the difference in $\Delta \chi^{2}$ obtained fitting the datasets of spectrum 1 and spectrum 2, when the real BAT spectrum is substituted with a BAT simulated spectrum. The $\chi^{2}$ difference is calculated for the two best-fit models: npex and npex+cyc.

on the adopted soft X-ray contribution, although we have just exploited the only two pointed observations with XRT and Suzaku available to date. The broad negative residuals are compatible with a resonant cyclotron absorption feature with an estimated significance of the detection at least at $\sim 4 \sigma$ level.

Similar features are often seen in the high energy spectra of sgHMXB. They are interpreted as cyclotron lines produced near the magnetic poles of the accretion-powered neutron star. These are due to resonant scattering processes of the X-rays by electrons whose kinetic energies are quantised into discrete Landau energy levels perpendicular to the B-field. Their detection is fundamental to the understanding of the accretion mechanisms onto the neutron star as it allows a direct measurement of the magnetic field of the neutron star (or at least a lower limit, depending on the height at which the resonant line absorption is effectively produced, see Nishimura 2011).

We consider here that the cyclotron absorption takes place at the poles of the neutron star, taking into account the relativistic gravitational redshift $E_{\mathrm{cyc}}^{\mathrm{obs}}=E_{\mathrm{cyc}}(1+z)^{-1}$, with

$(1+z)^{-1}=\left(1-\frac{2 G M_{\mathrm{X}}}{R_{\mathrm{X}} c^{2}}\right)^{0.5}$.

Assuming typical values of the NS mass and radius $\left(1.4 M_{\odot}\right.$ and $10 \mathrm{~km}$, respectively) and the observed value of the cyclotron line energy of $33 \pm 4 \mathrm{keV}$ (npex model for spectrum 1), this implies a magnetic field of $B_{\mathrm{NS}}(3.7 \pm 0.4) \times 10^{12}$ gauss at the surface of the NS.

The energy resolution of the spectra is insufficient to independently constrain the line energy, line width, and depth. We found that the line energy and depth could be relatively more tightly constrained than the line width. However, we also deterimined, in a series of fitting attempts, the best-fit models where a line width was frozen at $5 \mathrm{keV}$, and $8 \mathrm{keV}$ and $10 \mathrm{keV}$ to help us assess how sensitive the fitting was to variations in this parameter. We found in general that the $10 \mathrm{keV}$ guess always provided the lowest $\chi^{2}$, even though in most cases the improvements were marginal $\left(2<\Delta \chi^{2}<5\right)$, whereas the values of the other parameters were insensitive to the width choice.

This value represents, therefore, a first-order guess assumed after considering the emerging relations among the parameters that characterize the cyclotron line shapes. An empirical correlation between line energy and line width (Coburn et al. 2002) is, in fact, observed, where cyclotron lines detected between
$30 \mathrm{keV}$ and $40 \mathrm{keV}$ tend to also have significant line widths in the 5-10 keV range (e.g., in MX 0656-072, 4U 0352+309, XTE J1946+274, (see Coburn et al. 2002; McBride et al. 2006).

Another important empirical correlation concerns the cut-off energies of the continuum emission and the cyclotron line energy, where a correlation is observed with cut-off energies typically at half the value of the cyclotron energy. In this case, our results appear to agree with the general trend that is observed, with a cut-off energy at $\sim 15 \mathrm{keV}$ and the cyclotron energy at $\sim$ two times this value (Heindl et al. 2004).

Possible biases in this analysis are envisaged by the use of long-term time-averaged spectra for features that may show some variability. Some luminosity-dependent shifts of the cyclotron line position have clearly been observed in some sources (Mihara et al. 2004; Staubert et al. 2007; Nakajima et al. 2010). However, the large uncertainties in the present analysis estimates do not allow us to set this possibility into investigation. IGR J16493-4348 has never shown unusual periods of strong changes in its luminosity, nor any outbursting behaviour as in the case of X0331+53, where luminosity changed by a factor $\sim 200$ (Nakajima et al. 2010), so that we argue that possible shifts, if present, should be within our quoted error-bars. Moreover, we note that the cyclotron feature at $45 \mathrm{keV}$ of A0535+262 did not show any variations despite a change in luminosity of two orders of magnitude (Terada et al. 2006). Another source of bias concerns the possibility of continuum spectral changes that, when averaged, could result in spectral artifacts. The analysis of the soft X-ray spectrum from the XRT and Suzaku pointed observations are insufficient to test any variability pattern in the broadband spectral properties; use of simplified, phenomenological, models are a possible source of bias and a more physically based model should be employed to infer the physical properties of the accretion column environment (Becker \& Wolff 2007; Schönherr et al. 2007) and to more tightly constrain the shape of cyclotron resonance features.

\section{Conclusions}

We have presented spectral analysis in the high-energy X-ray band of IGR J16493-4348 using long-term survey data from BAT and ISGRI, complemented with pointed soft X-ray observations from XRT and Suzaku. The BAT spectrum is to date the highest $\mathrm{S} / \mathrm{N}$ spectrum of this source in the high-energy X-ray band. When the spectrum is complemented with soft X-ray data, 
a broad absorption feature is detected, irrespective of the broadband model used to fit the data. We have modelled the feature assuming that it is a CRSF. This interpretation is statistically well supported and the physical interpretation of the parameters agrees with that expected from the whole sample of HMXBs that display similar features. We estimated the significance level of the detection to be greater than $3.9 \sigma$ confidence level and the next generation of observatories dedicated to the high X-ray band (e.g., NuSTAR and Astro-H) will likely provide a more stringent constraint of its presence and a substantial improvement in the determination of its shape.

Acknowledgements. The authors acknowledge financial contribution from the agreement ASI-INAF I/009/10/0.

\section{References}

Barthelmy, S. D., Barbier, L. M., Cummings, J. R., et al. 2005, Space Sci. Rev., 120,143

Becker, P. A., \& Wolff, M. T. 2007, ApJ, 654, 435

Bird, A. J., Barlow, E. J., Bassani, L., et al. 2004, ApJ, 607, L33

Boldt, E. 1987, Phys. Rep., 146, 215

Burrows, D. N., Hill, J. E., Nousek, J. A., et al. 2005, Space Sci. Rev., 120, 165

Coburn, W., Heindl, W. A., Rothschild, R. E., et al. 2002, ApJ, 580, 394

Corbet, R. H. D. 1984, A\&A, 141, 91

Corbet, R. H. D., Pearlman, A. B., \& Pottschmidt, K. 2010, The Astronomer's Telegram, 2766, 1
Cusumano, G., La Parola, V., Romano, P., et al. 2010a, MNRAS, 406, L16 Cusumano, G., La Parola, V., Segreto, A., et al. 2010b, A\&A, 510, A48 Fukazawa, Y., Mizuno, T., Watanabe, S., et al. 2009, PASJ, 61, 17 Gehrels, N., Chincarini, G., Giommi, P., et al. 2004, ApJ, 611, 1005

Grebenev, S. A., Bird, A. J., Molkov, S. V., et al. 2005, The Astronomer's Telegram, 457, 1

Heindl, W. A., Rothschild, R. E., Coburn, W., et al. 2004, in AIP Conf. Ser., Vol. 714, X-ray Timing 2003: Rossi and Beyond, ed. P. Kaaret, F. K. Lamb, \& J. H. Swank, 323-330

Hill, J., Burrows, D., Nousek, J., et al. 2004, APS Meeting Abstracts, 10005

Hill, A. B., Dean, A. J., Landi, R., et al. 2008, MNRAS, 385, 423

Koyama, K., Tsunemi, H., Dotani, T., et al. 2007, PASJ, 59, 23

Kuiper, L., Jonker, P., Hermsen, W., \& O'Brien, K. 2005, The Astronomer's Telegram, 654, 1

Makishima, K., Mihara, T., Ishida, M., et al. 1990, ApJ, 365, L59

Makishima, K., Mihara, T., Nagase, F., \& Tanaka, Y. 1999, ApJ, 525, 978

McBride, V. A., Wilms, J., Coe, M. J., et al. 2006, A\&A, 451, 267

Mihara, T., Makishima, K., \& Nagase, F. 2004, ApJ, 610, 390

Mitsuda, K., Bautz, M., Inoue, H., et al. 2007, PASJ, 59, 1

Morris, D. C., Smith, R. K., Markwardt, C. B., et al. 2009, ApJ, 699, 892

Nakajima, M., Mihara, T., \& Makishima, K. 2010, ApJ, 710, 1755

Nespoli, E., Fabregat, J., \& Mennickent, R. E. 2010, A\&A, 516, A106

Nishimura, O. 2011, ApJ, 730, 106

Reig, P. 2011, Ap\&SS, 332, 1

Schönherr, G., Wilms, J., Kretschmar, P., et al. 2007, A\&A, 472, 353

Segreto, A., Cusumano, G., Ferrigno, C., et al. 2010, A\&A, 510, A47

Staubert, R., Shakura, N. I., Postnov, K., et al. 2007, A\&A, 465, L25

Suchy, S., Pottschmidt, K., Rothschild, R. E., et al. 2011, ApJ, 733, 15

Takahashi, T., Abe, K., Endo, M., et al. 2007, PASJ, 59, 35

Terada, Y., Mihara, T., Nakajima, M., et al. 2006, ApJ, 648, L139 